\title{
Simplified Estimation of Train Resistance Parameters: Full Scale Experimental Tests and Analysis
}

\author{
C. Somaschini, D. Rocchi, G. Tomasini and P. Schito \\ Department of Mechanical Engineering \\ Politecnico di Milano \\ Italy
}

\begin{abstract}
A CEN standard (EN 14067-4, 2005) describes the methodologies for the assessment of the running resistance of railway vehicles starting from full-scale test measurements. According to this standard, the speed dependent terms of the equation of Davis [1] have to be determined by means of coasting tests. In this paper, a new method to estimate the running resistance coefficients from a full-scale coasting test is proposed and compared with the two methods proposed in the CEN standard (the regression method and the speed history identification method). The main advantage of this new method is that it does not require the railway line characteristics to be known and it will be shown that the new method is able to evaluate the coefficients with an accuracy equivalent to that of the other methods considered.
\end{abstract}

Keywords: train running resistance, full scale tests, regression method, speed history method, coasting tests.

\section{Introduction}

For determining the running resistance force $F_{r}$, the most adopted formula is the second degree polynomial with respect to the speed developed in 1926 by Davis [1] and written as:

$$
F_{r}=A m+B v+C v^{2}
$$

In Equation (1), term $A$ contains resistances which do not depend on the vehicle speed but are function of the mass. The constant $B$ accounts for the resistance associated to the mechanical resistances and the HVAC while the coefficient $C$ represents the resistances that are proportional to the square of the speed and are connected to the aerodynamic resistance due to the air pressure and friction. 
In the past, run-down tests were performed for the determination of these coefficients: coasting tests, by measuring the speed variation versus time, and constant speed tests, by measuring the tractive effort necessary to maintain a constant velocity.

Running resistance coefficients of different trains are reported in [2] for the Shinkansen, in [3] for the TGV-PSE and in [4] for the ICE/V. Moreover, different testing methods and running resistance applied to the loco-hauled Eurofima coaches are presented in [5].

On the other hand, because these tests are very costly, in the past, different empirical equations for the estimation of the resistance of specific trains were developed. An overview of the methods adopted by the main national railways is reported in [6] (up to the 2000 year): in this paper, a tool for calculating train resistance, able to account for also the contributions to the running resistance of various features of the architecture of the train, is compared with the results of other equations for calculating train resistance.

More recently (2007), Lukaszewicz proposes a method which allows to determine train running resistance coefficients from full-scale coasting tests by measuring only train speed and position ([7]): the resistance is determined by calculating the change in kinetic and potential energy of a coasting train between successive measurement positions. Using this method, in [8] the same author presents experimental results for determining the running resistance for different trains and the influence of variables such as speed, number of axles, number of coaches, axle load, track type and train length.

Since 2005, a CEN standard ([9]) describes the methodologies for the assessment of the coefficients of the Davis' formula (Equation (1)) starting from full-scale test measurements: on other approaches, based on predictive formula, numerical simulations and reduced-scale tests, there are no agreed methods available.

According to the CEN standard, the determination of the speed dependent terms ( $B$ and $C$ ) of Equation (1) is carried out by means of coasting tests. For the $A$ term, a special test is needed, consisting on hauling the train at very low speed.

In the CEN standard, two different post-test data treatments to obtain the resistance coefficients $B$ and $C$ from a coasting test are described: the regression method and the speed history identification method. The first consists in interpolating all the experimental data available while the second is based on the integration of the equation of motion. Both these methods require to know very well the characteristics of the test section (slopes and curve radii).

In this paper, the standard methods for the identification of the Davis coefficients are compared with the new methods. In particular, it will be shown that the three coefficients of the Davis formula can be estimated only by two tests, that are the coasting test at very low speed over a high sloping section (without the need of performing the hauling test) and the coasting test starting from the maximum train speed.

Moreover, a new method (POLIMI regression method) is proposed to identify the resistance coefficients $B$ and $C$ from the coasting tests. The main advantage of this method is that it does not require to know the railway line characteristics and the coefficient $A$ of the Davis equation. 
Starting from the experimental full-scale tests, rescaled for a generic ideal train (characterised by a mass of 450 tons), the whole procedure for the determination of the running resistance coefficients is described. $A$ comparison between the results obtained with the different methods for the estimation of the coefficients $B$ and $C$ of the Davis' equation will be presented and analysed.

\section{Description of the problem}

The equation of motion of the train in its running direction is:

$$
\left(m+J^{*} / R^{2}\right) a=\sum F_{i}
$$

where $m$ is the total mass of the train, $J^{*}$ is the generalised moment of inertia of the rotating masses, $R$ is the wheel radius, $a$ the acceleration of the train and $\sum F_{i}$ is the sum of all the forces resisting to the motion of the train.

Gathering the terms on the left and setting out the one on the right, it's possible to rewrite this equation as ([9]):

$$
m k a=-\left(F_{r}+F_{p}+F_{c}\right)
$$

The Equation (3) correlates the "mass-factor" $k$ to the resistance force $F_{r}$, the gravitational forces $F_{p}$ (depending on the slope of the track) and friction $F_{c}$ (function of the radius of the curves, considered negligible in a straight line).

Knowing the data of the track (slope $\alpha$, from which calculate $i=\sin \alpha$, and radius of curvature $R_{c}$ in meters), it is possible to approximate $F_{p}$ as:

$$
F_{p}=m g i
$$

and $F_{c}$ by means of the empirical formula:

$$
F_{c}=m g \frac{0.8}{R_{c}}
$$

while the resistance force is an unknown parameter, a priori. As described by the standard, this force can be approximated using the Davis formula that correlates this term with a second order function of the speed of train, as reported in Equation (1), where $A, B$ and $C$ are three unknown coefficients.

It is therefore possible to rewrite Equation (3) as:

$$
m \boldsymbol{k} a=-\left(\boldsymbol{A} m+\boldsymbol{B} v+\boldsymbol{C} v^{2}+m g i+m g \frac{0.8}{R_{c}}\right)
$$

where the terms in bold are the four constant coefficients which have to be estimated with experimental tests.

\section{Test characteristics and results}

Two types of experimental tests were carried out for the determination of the constant coefficients: 
- coasting tests uphill and downhill on a straight track section with constant slope at low speed, for the determination of the mass factor $\mathrm{k}$;

- coasting tests over a section with different starting speeds (from the maximum train speed) for the determination of the coefficients of the Davis formula ( $A, B$ and $C$ ).

All the tests were carried out on railroad lines whose characteristics were known. During the experimental runs, the only parameter measured onboard was the position of the train by means of an encoder; the speed and the acceleration of the train were obtained deriving the position with respect to the time.

On the other hand, several anemometers and weather station were placed trackside in order to measure the environmental conditions during the tests. In this way, it was possible to remove from the analysis the runs characterized by a mean wind speed higher than $2 \mathrm{~m} / \mathrm{s}$; all the other tests were considered valid and were analyzed without considering the wind.

\subsection{Mass factor}

The test for the determination of the mass factor $k$ is required to define the contribution of the inertia of the rotating masses of the train ([9]).

The experimental test was carried out on a track section with constant slope equal to $\alpha=22 \%$. This test consists in running the train to a speed $\mathrm{v}(\mathrm{v} \leq 30 \mathrm{~km} / \mathrm{h})$ at the start point $S$ and then proceeding in coasting until reaching zero speed (turning point T). Subsequently the train, always in coasting, due to the gravitational force, retraces the same stretch $\Delta s$ downhill until the point of departure S.

Making an energy balance on the stretch $\Delta s$ uphill and downhill:

$$
\left\{\begin{array}{l}
\frac{1}{2} m k v_{S 1}{ }^{2}=m g i \Delta s+\int_{S}^{T} F_{r} d s \\
m g i \Delta s=\frac{1}{2} m k v_{S 2}{ }^{2}+\int_{T}^{S} F_{r} d s
\end{array}\right.
$$

and supposing that the integral of the resistance force is almost equal in the two runs:

$$
\int_{S}^{T} F_{r} d s \cong \int_{T}^{S} F_{r} d s
$$

By combining Equation (7), it is possible to estimate the mass factor $k$ as:

$$
k=\frac{4 g i \Delta s}{v_{S 1}{ }^{2}+v_{S 2}{ }^{2}}
$$

where $v_{S 1}$ and $v_{S 2}$ are the train speed at the starting point $\mathrm{S}$, respectively at the entrance and the exit of the test section.

The reference speed of the test, intended as the initial speed, is equal to $v_{r e f}=$ $30 \mathrm{~km} / \mathrm{h}$ and the same test was performed for three different initial speeds:

- $v_{S 1}=v_{\text {ref }}$;

- $v_{S 1}=0.8 v_{\text {ref }}$;

- $v_{S 1}=0.6 v_{\text {ref }}$; 
with nine repetitions for each speed in order to check the repeatability of the results. Table 1 shows the results for each initial speed tested.

\begin{tabular}{c|ccccc}
\hline Test speed & $\begin{array}{c}\boldsymbol{v}_{\boldsymbol{1}} \\
{[\mathbf{k m} / \mathbf{h}]}\end{array}$ & $\begin{array}{c}\boldsymbol{v}_{\boldsymbol{2}} \\
{[\mathbf{k m} / \mathbf{h}]}\end{array}$ & $\begin{array}{c}\Delta \boldsymbol{s} \\
{[\mathbf{m}]}\end{array}$ & $\begin{array}{c}\boldsymbol{k} \\
\text { mean }\end{array}$ & $\begin{array}{c}\boldsymbol{\sigma}_{\boldsymbol{k}} \\
\text { std }\end{array}$ \\
\hline $\boldsymbol{v}_{\boldsymbol{S 1}}=\boldsymbol{v}_{\boldsymbol{r e f}}$ & 30.00 & 28.9 & 160.4 & 1.0385 & $0.58 \mathrm{e}^{-3}$ \\
$\boldsymbol{v}_{\boldsymbol{S 1}}=\mathbf{0 . 8} \boldsymbol{v}_{\boldsymbol{r e f}}$ & 24.00 & 23.1 & 102.8 & 1.0394 & $0.68 \mathrm{e}^{-3}$ \\
$\boldsymbol{v}_{\boldsymbol{S 1}}=\mathbf{0 . 6} \boldsymbol{v}_{\text {ref }}$ & 18.00 & 17.3 & 57.6 & 1.0395 & $0.57 \mathrm{e}^{-3}$ \\
\hline
\end{tabular}

Table 1: Initial and final train speeds, space covered and estimate of the mass factor $k$.

The results have a very small dispersion $(\sigma=7 \% 0)$ proving the validity of the method used. Averaging all the results, we obtained a mass factor of 1.0392 and a corresponding generalized mass of the rotating parts of about $17000 \mathrm{~kg}$.

\subsection{Coefficient A}

As regard with the coefficient $A$ of the equation of Davis, the standard prescribes a particular test called 'train hauling procedure' that consists of pulling the train at constant speed using a windlass. On the other hand, in this paper an alternative procedure to evaluate this parameter is proposed starting from the tests performed for the determination of the mass factor (at low speed, uphill and downhill on a straight track section) as shown below.

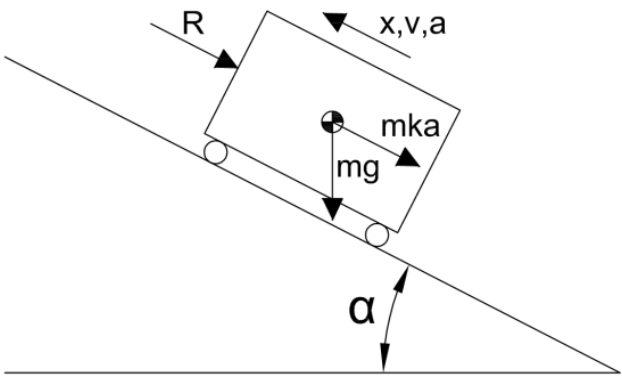

(a)

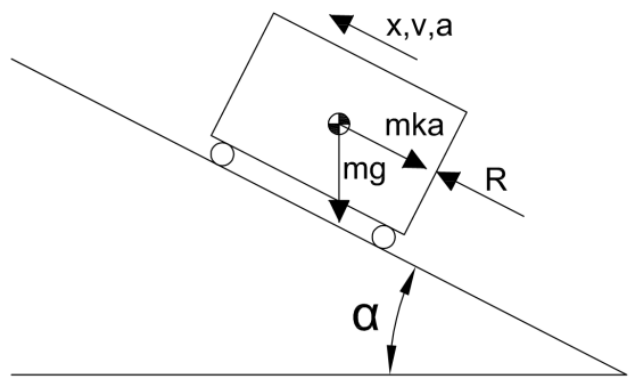

(b)

Figure 1: Forces acting on the train for uphill (a) and downhill runs (b) (same reference system for dispacement $x$, velocity $v$ and acceleration $a$ ).

Considering the whole test uphill and downhill (and the reference system shown in Figure 1), it is possible to express the equations of motion of the two movements as:

$$
\left\{\begin{array}{l}
m k a_{1}=-\left(A+B\left|v_{1}\right|+C v_{1}^{2}\right)-m g i \\
m k a_{2}=\left(A+B\left|v_{2}\right|+C v_{2}^{2}\right)-m g i
\end{array}\right.
$$


Considering only the last part of the climb at very low speed $(\mathrm{v}<10 \mathrm{~km} / \mathrm{h})$, before changing direction, and the subsequent downhill section at the same speed, it is possible to neglect the effect of the terms given by coefficients $B$ and $C$.

The system of equations (10), ignoring the terms depending on the speed, becomes:

$$
\left\{\begin{array}{l}
k a_{1}=-\hat{A}-g i \\
k a_{2}=+\hat{A}-g i
\end{array}\right.
$$

having indicated by $\hat{A}$ the estimate of the coefficient $A$ obtained by this method. Therefore, adding and subtracting the two equations leads to:

$$
\left\{\begin{array}{l}
k\left(a_{1}+a_{2}\right)=-2 g i \\
k\left(a_{1}-a_{2}\right)=-2 \hat{A}
\end{array}\right.
$$

From which it is possible to get to:

$$
\left\{\begin{array}{l}
i=-\frac{k\left(a_{1}+a_{2}\right)}{2 g} \\
\hat{A}=-\frac{k\left(a_{1}-a_{2}\right)}{2}
\end{array}\right.
$$

It can be noted that, although the term $i$ is known, this is not imposed as an input but it is a result of the calculation. The comparison between $i$ and the nominal value of the slope (22\%) is therefore an index of reliability and correctness of the estimate.

Ignoring the terms depending on the speed, in order to calculate the accelerations in the two stretches it is possible to interpolate the trend of the speeds with two straight lines as shown, by way of example, in Figure 2.

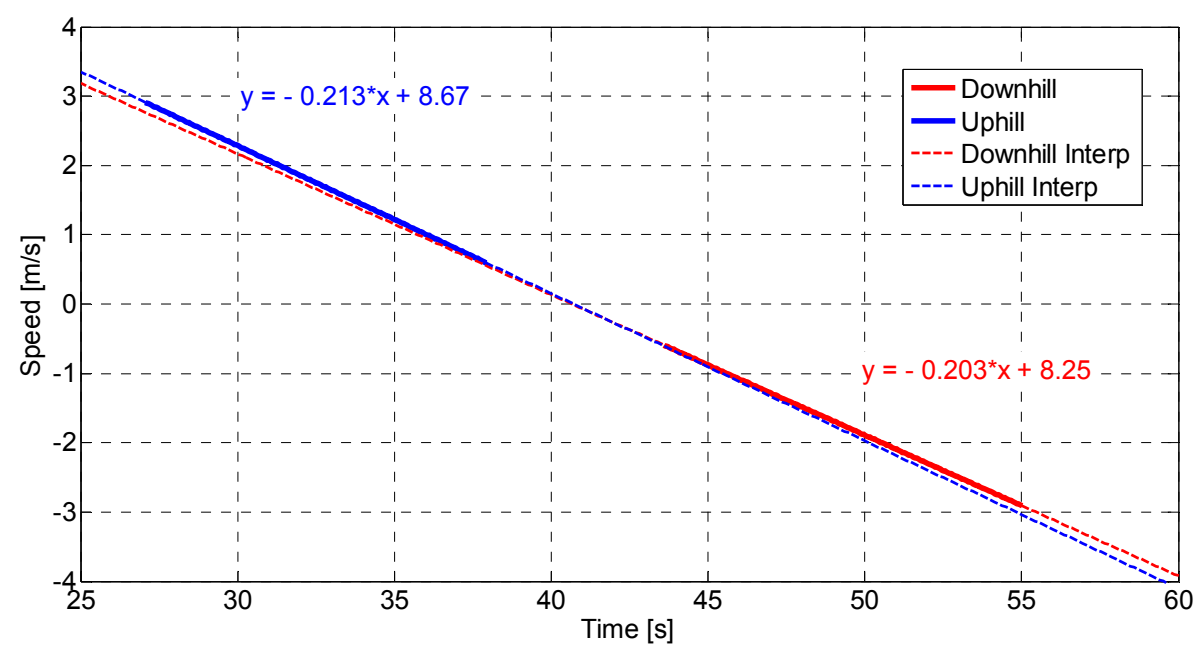

Figure 2: Example of interpolation of the speed in correspondence with the change of direction. 
Isolating the angular coefficient:

$$
\begin{aligned}
& a_{1}=-0.213 \mathrm{~m} / \mathrm{s}^{2} \\
& a_{2}=-0.203 \mathrm{~m} / \mathrm{s}^{2}
\end{aligned}
$$

From which, for the example of Figure 2, we estimated:

$$
\left\{\begin{array}{l}
i=0.02204 \\
\hat{A}=5.16 e^{-3}
\end{array}\right.
$$

By repeating the procedure for all the nine tests, the values shown in Table 2 are calculated.

\begin{tabular}{c|cccc}
\hline Test & $\begin{array}{c}\boldsymbol{a}_{\mathbf{1}} \\
{\left[\mathbf{m} / \mathbf{s}^{2}\right]}\end{array}$ & $\begin{array}{c}\boldsymbol{a}_{2} \\
{\left[\mathbf{m} / \mathbf{s}^{2}\right]}\end{array}$ & $\begin{array}{c}\boldsymbol{i} \\
{[\% \mathbf{0}]}\end{array}$ & $\begin{array}{c}\hat{A} \\
{[\mathbf{N} / \mathbf{k g}]}\end{array}$ \\
\hline $\mathbf{1}$ & -0.2128 & -0.2029 & 0.0220 & $5.16 \mathrm{e}^{-3}$ \\
$\mathbf{2}$ & -0.2127 & -0.2026 & 0.0220 & $5.29 \mathrm{e}^{-3}$ \\
$\mathbf{3}$ & -0.2132 & -0.2030 & 0.0220 & $5.27 \mathrm{e}^{-3}$ \\
$\mathbf{4}$ & -0.2132 & -0.2030 & 0.0220 & $5.31 \mathrm{e}^{-3}$ \\
$\mathbf{5}$ & -0.2117 & -0.2026 & 0.0219 & $4.72 \mathrm{e}^{-3}$ \\
$\mathbf{6}$ & -0.2131 & -0.2030 & 0.0220 & $5.25 \mathrm{e}^{-3}$ \\
$\mathbf{7}$ & -0.2129 & -0.2027 & 0.0220 & $5.31 \mathrm{e}^{-3}$ \\
$\mathbf{8}$ & -0.2131 & -0.2030 & 0.0220 & $5.26 \mathrm{e}^{-3}$ \\
$\mathbf{9}$ & -0.2125 & -0.2024 & 0.0220 & $5.27 \mathrm{e}^{-3}$ \\
Mean & & & & $\mathbf{5 . 2 7} \mathrm{e}^{-3}$ \\
\hline
\end{tabular}

Table 2: Acceleration of the train near the turning point in the runs. The average is calculated by excluding the results with $i \neq 22$ highlighted in grey.

Actually, not taking into account the terms speed-depending, $\hat{A}$ overestimate the value of the coefficient $A$ but the goodness of the valuation and how to evaluate a more accurate value of $A$ will be show in section 3.3.1.

\subsection{Coefficients B and C}

The coefficients $B$ and $C$ of the equation of Davis were calculated using seven experimental runs with different speed ranges (see Table 3). The train entered the test section in coasting with the desired speed and with any type of mechanical brake, electrical and magnetic disabled. From the position measured by the encoder it is possible to know the absolute position of the train and therefore the instantaneous values of slope and curvature radius while the speed and the acceleration of the train were obtained deriving the position with respect to the time (see Figure 3). 

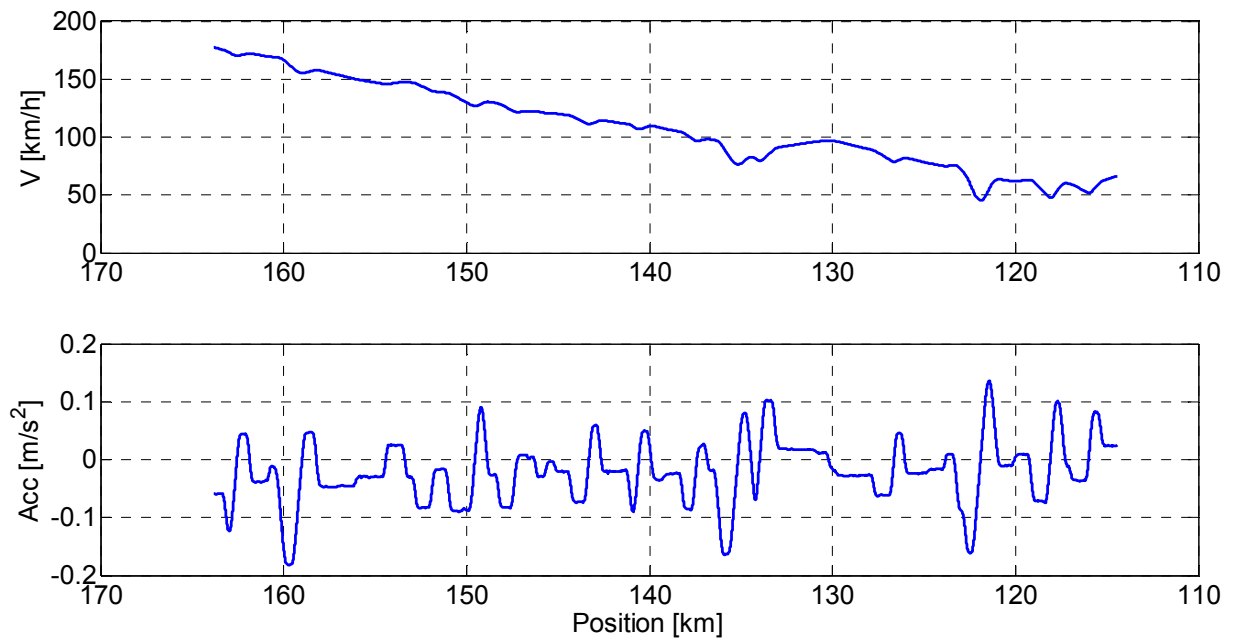

Figure 3: Example of experimental results in terms of speed and acceleration for RUN \#1.

\begin{tabular}{|c|c|c|c|c|}
\hline \# RUNS & DIRECTION & $\begin{array}{c}\mathbf{V}_{\text {MIN }} \\
{[\mathbf{k m} / \mathbf{h}]}\end{array}$ & $\begin{array}{c}\mathbf{V}_{\text {MAX }} \\
{[\mathbf{k m} / \mathbf{h}]}\end{array}$ & $\begin{array}{c}\mathbf{\rho} \\
{\left[\mathbf{k g} / \mathbf{m}^{\mathbf{3}}\right]}\end{array}$ \\
\hline $\mathbf{1}$ & 1 & 40 & 180 & 1.239 \\
\hline $\mathbf{2}$ & 2 & 60 & 180 & 1.235 \\
\hline $\mathbf{3}$ & 1 & 60 & 200 & 1.232 \\
\hline $\mathbf{4}$ & 1 & 120 & 270 & 1.253 \\
\hline $\mathbf{5}$ & 2 & 150 & 290 & 1.253 \\
\hline $\mathbf{6}$ & 1 & 40 & 130 & 1.254 \\
\hline $\mathbf{7}$ & 2 & 140 & 300 & 1.254 \\
\hline
\end{tabular}

Table 3: List of the experimental runs

Looking at the equation (6), now the only unknown parameters are the terms $B$ and $C$, which are estimable in different ways. As prescribe by the standard, the most simple way, if the characteristics of the line are known, is to gather all the runs and interpolate the resulting curve $F_{r}(v)$ (regression method). This is the easiest but also the least accurate method because it does not take into account for example possible differences in the air density ${ }^{1}$ values between different days. On the other hand, according to the CEN standard, an alternative method is the speed history identification method: with this approach, the experimental runs can be analysed one by one integrating the equation of motion, estimating $B$ and $C$ minimising the error with respect to the experimental data. The problems in this case is that the results depend on the speed range analysed, which means that the dispersion of the results is much higher. In order to obtain more accurate estimates, it is possible to merge the two methods presented in the standard, interpolating the curve $F(v)$ of the numerical simulation instead of the experimental data (Regression method on the

\footnotetext{
${ }^{1}$ Since the term $C v^{2}$ is due to the dynamic pressure, it can be rewrites as $C v^{2} \cong \bar{C} 0.5 \rho S v^{2}$ where $\rho$ is the air density while $S$ is a reference section of the train.
} 
speed history identification method data): the advantage of this procedure is that all the data can be included, accounting for the variations of radius, slope and air density.

The results obtained in this way are accurate but the prerogative is to know the value of $A$ and the exact values of the characteristics of the line (first of all the slope). This is not always possible and inaccurate input data could bring to big errors.

For this reason, a new method (POLIMI differential method) able to estimate the coefficients $B$ and $C$ without knowing the property of the railroad line nor the coefficient $A$, have been devised.

\subsubsection{Regression method}

Rewriting equation (6) as:

$$
F(v)=-\left(B v+C v^{2}\right)=m k a+A m+m g i+m g \frac{0.8}{R_{c}}
$$

and merging the data of all the test runs, it is possible to trace the trend of the force $\mathrm{F}$ as a function of the speed $v$ as shown in Figure 4. Note that, in order to decrease the margin of error due to the corrections of $F_{p}$ and $F_{c}$, the study has been limited only to the straight sections with a slope constant and lower than $5 \%$.

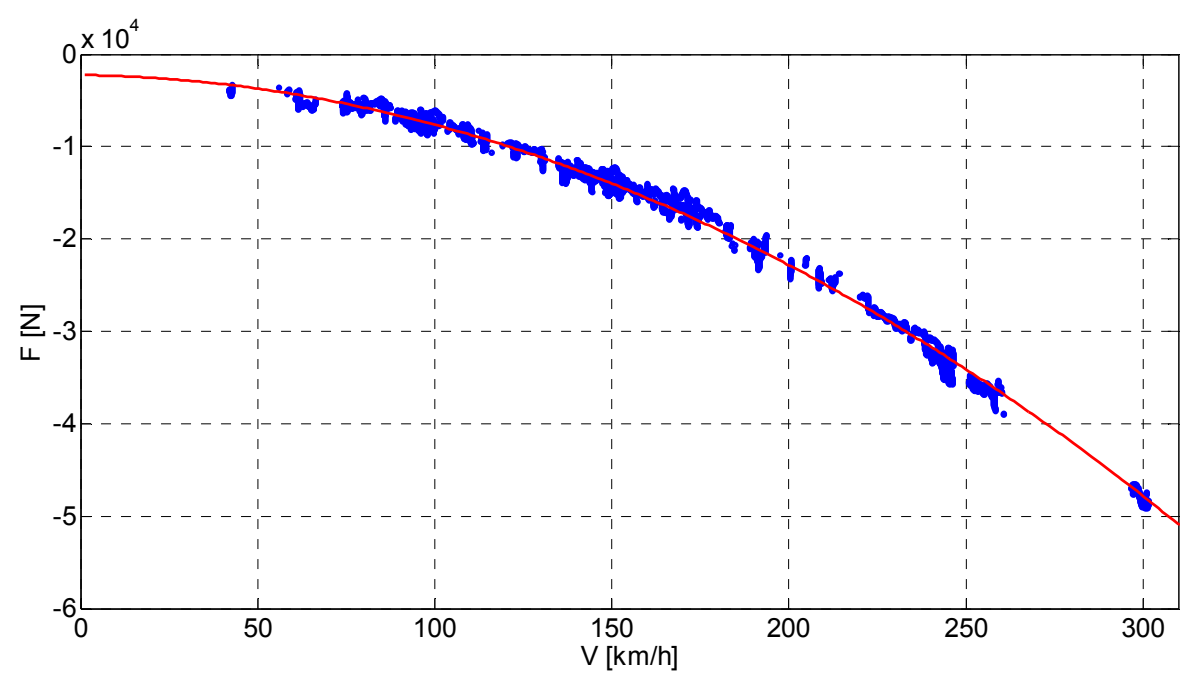

Figure 4: Experimental results of resistance force as a function of the speed (dotted line) and the best interpolating curve (solid line).

Therefore, interpolating the experimental curve, the results summarized in Table 4 were obtained. In particular, the first row refers to the case in which all the three parameters were left free, while, the second one shows the results obtained fitting the experimental data with the coefficient $A$ imposed. These results were achieved through iterative steps comparing the value of $\hat{A}$ (obtained using the method 
proposed in 3.1 on numerical simulations performed with the estimated coefficients) with the one previously estimated by experimental data.

\begin{tabular}{|c|c|c|c|c|c|}
\hline \# sim & $\begin{array}{c}\text { A } \\
{[\mathbf{N} / \mathbf{k g}]}\end{array}$ & $\begin{array}{c}\text { B } \\
{[\mathrm{Ns} / \mathrm{m}]}\end{array}$ & $\begin{array}{c}\mathbf{C} \\
{\left[\mathrm{Ns}^{2} / \mathbf{m}^{2}\right]}\end{array}$ & $\begin{array}{c}\text { Error } \\
{[\mathbf{N}]}\end{array}$ & $\begin{array}{c}\widehat{A} \\
{[\mathbf{N} / \mathbf{k g}]}\end{array}$ \\
\hline $\begin{array}{c}\text { A, B and } \\
\text { C free }\end{array}$ & $6.06 \mathrm{e}^{-3}$ & 0.00 & 6.519 & 866.94 & $6.11 \mathrm{e}^{-3}$ \\
\hline A fixed & $5.14 \mathrm{e}^{-3}$ & 17.04 & 6.357 & 871.23 & $\mathbf{5 . 2 7} \mathrm{e}^{-3}$ \\
\hline
\end{tabular}

Table 4: Results of the various optimizations of the coefficients $\mathrm{B}$ and $\mathrm{C}$ and respective values of the estimate $\hat{A}$.

The mistake made in the optimization (calculated as the square error between the experimental data and the interpolation) is minimal in the first case while it is slightly higher in the other three cases. Considering also the value of $\hat{A}$ (obtained from the simulations performed with the estimated coefficients), it is however possible to say that the couple of coefficients that best approximates the experimental curve is the one of the second raw and the Davis resulting equation is thus:

$$
F_{r}(m, v)=-5.138 \cdot 10^{-3} m-17.04 \cdot v-6.357 \cdot v^{2}
$$

Where the mass $m$ and the velocity $v$ are expressed respectively in $[\mathrm{kg}]$ and $[\mathrm{m} / \mathrm{s}]$ to obtain a resisting force $F_{r}$ in [N].

\subsubsection{Speed history identification method}

We have numerically simulated all the seven runs, obtaining optimised coefficients $B$ and $C$ for each run. Even in this case, it is neglected any aerodynamic effect due to the presence of wind; on the other hand, the curves and slopes have been considered as required by the standards.

In Figure 5, an example of the results obtained using the data of the first run is presented: speed trend as a function of space compared with the experimental data. To facilitate comparison, in Figure 5, also the errors committed are reported in terms of distance travelled and speed.

Finally, in Table 5 all the results obtained by the numerical simulations are summarized considering the term A constant and equal to $5.14 \mathrm{e}^{-3}$.

The results show a big dispersion, especially the coefficient $B$, probably because each runs has a different speed range, which means that the coefficients $\mathrm{B}$ and $C$ have different weights. This is the main reason that prompted us to combine this method with the previous one in order to try to make a weighted average of the results. 

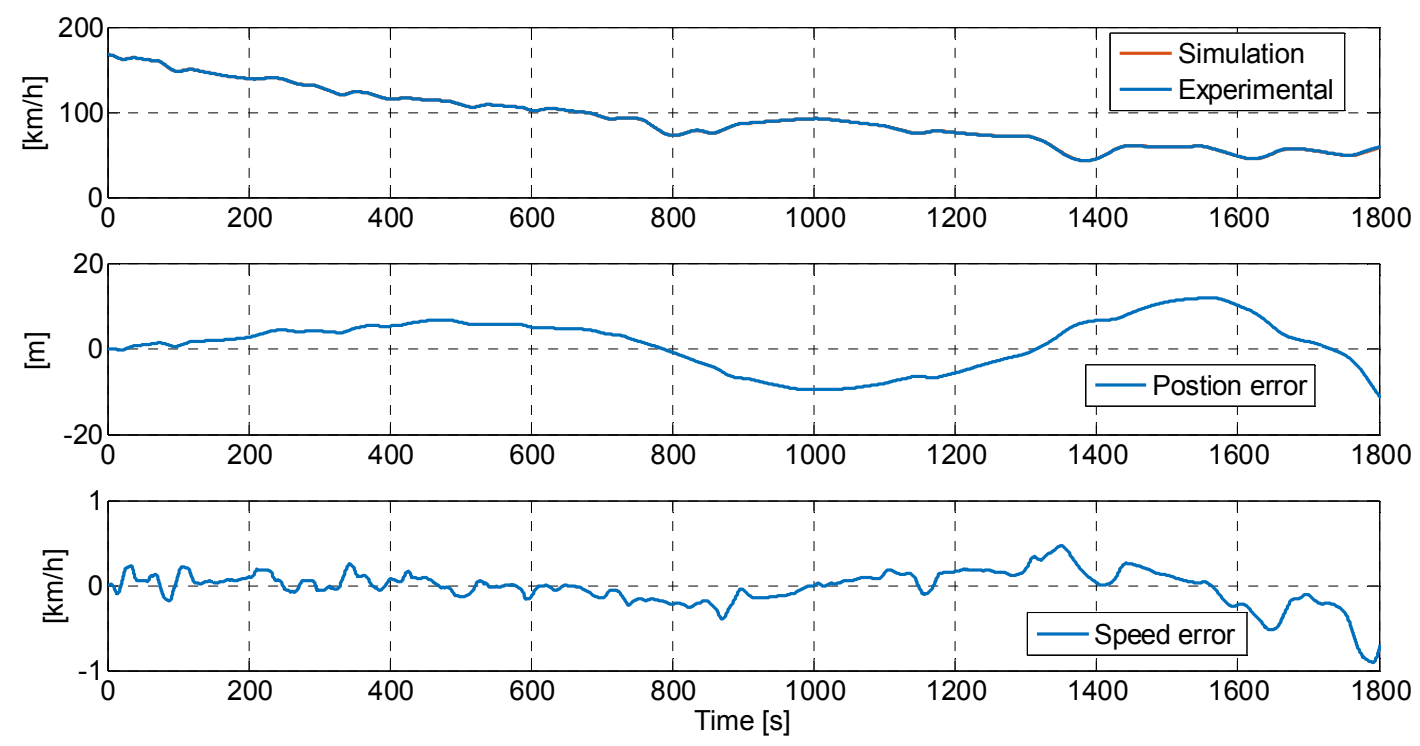

Figure 5: Comparison between the experimental data and the estimate of the optimised numerical simulation.

\begin{tabular}{|c|c|c|c|c|c|c|c|}
\hline \# RUNS & DIRECTION & $\begin{array}{c}\mathbf{V}_{\text {MIN }} \\
{[\mathbf{k m} / \mathbf{h}]}\end{array}$ & $\begin{array}{c}\mathbf{V}_{\text {MAX }} \\
{[\mathbf{k m} / \mathbf{h}]}\end{array}$ & $\begin{array}{c}\mathbf{B} \\
{[\mathbf{N s} / \mathbf{m}]}\end{array}$ & $\begin{array}{c}\mathbf{C} \\
{\left[\mathbf{N s}^{\mathbf{2}} / \mathbf{m}^{\mathbf{2}}\right]}\end{array}$ & $\begin{array}{c}\text { Max } \\
\mathbf{E r r} \mathbf{x} \\
{[\mathbf{m}]}\end{array}$ & $\begin{array}{c}\text { Max } \\
\mathbf{E r r} \mathbf{~} \\
{[\mathbf{k m} / \mathbf{h}]}\end{array}$ \\
\hline $\mathbf{1}$ & 1 & 40 & 180 & 42.56 & 5.53 & 21 & 0.9 \\
\hline $\mathbf{2}$ & 2 & 60 & 180 & 0.00 & 6.84 & 37 & 1.5 \\
\hline $\mathbf{3}$ & 1 & 60 & 200 & 18.57 & 6.15 & 17 & 1.1 \\
\hline $\mathbf{4}$ & 1 & 120 & 270 & 4.70 & 6.43 & 11 & 0.4 \\
\hline $\mathbf{5}$ & 2 & 150 & 290 & 38.86 & 6.14 & 3 & 0.3 \\
\hline $\mathbf{6}$ & 1 & 40 & 130 & 17.76 & 6.50 & 4 & 0.8 \\
\hline $\mathbf{7}$ & 2 & 140 & 300 & 37.85 & 6.20 & 3 & 0.3 \\
\hline MEAN & & & & $\mathbf{2 2 . 9 0}$ & $\mathbf{6 . 2 6}$ & & \\
\hline STD & & & & $\mathbf{1 5 . 8 8}$ & $\mathbf{0 . 3 7}$ & & \\
\hline
\end{tabular}

Table 5: Simulations results and respective errors. 


\subsubsection{Regression method on the speed history identification method data}

The idea is to apply the regression method not using the experimental data but numerical data set obtained with the optimized simulations presented above. In this way it possible to consider all the runs (without excluding the slope and the radius changes) and also to account for the variations of the air density that were measured.

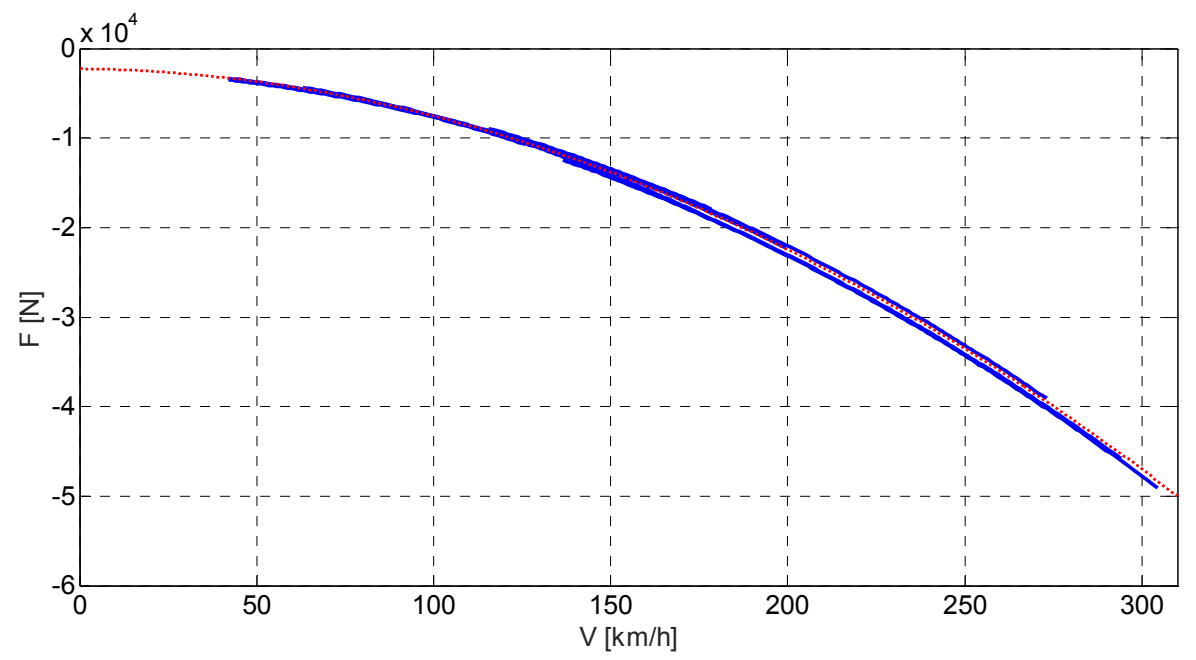

Figure 6: Numerical results of the resistance force as a function of the speed (solid lines) and the best interpolating curve (dotted line).

The results optained are showed in Figure 6 where all the numerical curves $F(v)$ (solid lines) and the best-fitting interpolation (dotted line) characterized by the coefficients $B$ and $C$ of 18.64 and 6.22 respectively are reported.

\subsubsection{The POLIMI differential method}

In all of the above methods, knowing the exact slope in each point of the railways line is essential in order to subtract from the total force the gravity term. Furthermore, the coefficient A has to be known even though it is the least important at high speed.

Practically this is not always possible and, furthermore, the gravity term of Equation (1) is the larger. This means that a small error in the slope or in the position brings to a big error in the evaluation of the aerodynamic force. By way of example, in Figure 7 the estimate of the resistance force of run 4 is reported; the big fluctuations due to these problems are clearly visible. 


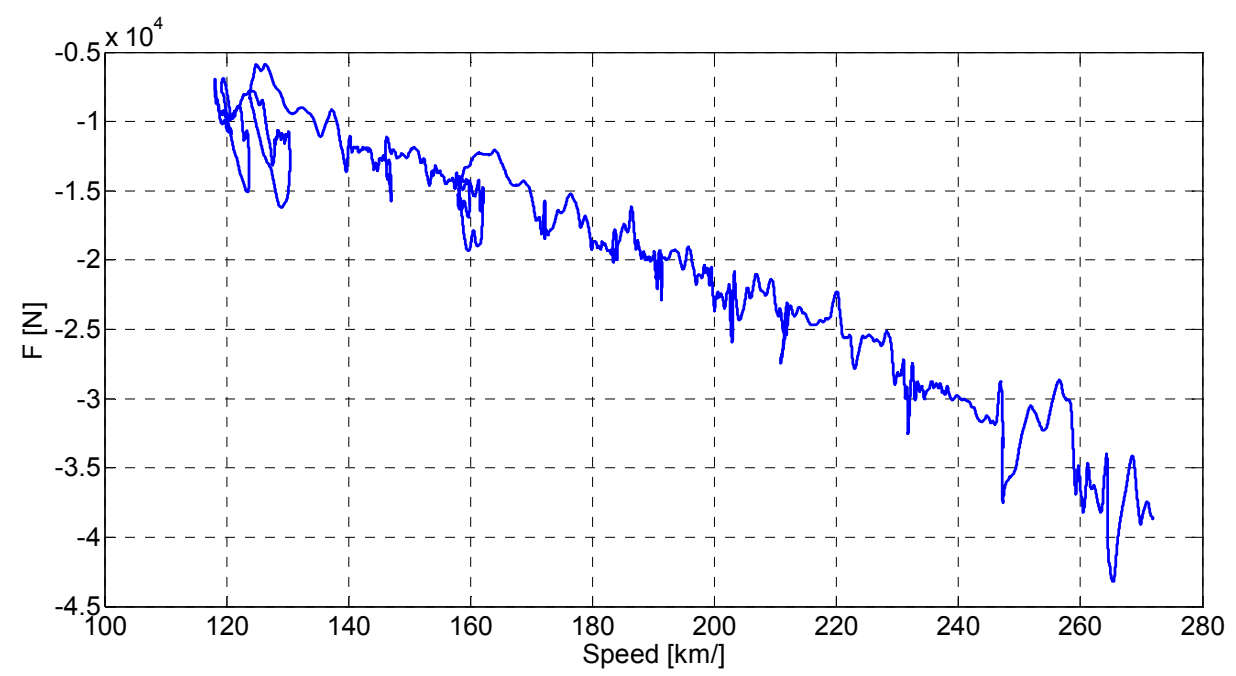

Figure 7: Experimental results of resistance force as a function of the speed in run 4 case.

The idea at the base of the presented method is that the forces due to gravity and curves in each point of the track are not function of the travelling speed. This means that if we carry out two tests on the same track at different speeds, in each position the equation of motion is written as:

$$
\left\{\begin{array}{l}
m k a_{1}=-\left(A m+B v_{1}+C v_{1}^{2}\right)-m g i-F_{c} \\
m k a_{2}=-\left(A m+B v_{2}+C v_{2}^{2}\right)-m g i-F_{C}
\end{array}\right.
$$

By subtracting the two equations (15), it is possible to obtain a new equation without the constant terms:

$$
m k\left(a_{1}-a_{2}\right)=-B\left(v_{1}-v_{2}\right)-C\left(v_{1}{ }^{2}-v_{2}{ }^{2}\right)
$$

Replacing the differential terms with:

$$
\begin{aligned}
a_{d} & =\left(a_{1}-a_{2}\right) \\
v_{d} & =\left(v_{1}-v_{2}\right) \\
v_{d_{-} q}^{2} & =\left(v_{1}^{2}-v_{2}{ }^{2}\right)
\end{aligned}
$$

the equation can be rewritten as:

$$
F_{d}=m k a_{d}=-B v_{d}-C v_{d_{-} q}^{2}
$$

that is the equation of a plane as a function of the variables $v_{d}$ and $v_{d_{-} q}^{2}$. In Figure 8, the results obtained using the data of test 1 and 4 are shown; comparing the curve with the one presented in Figure 7 it is clear that the oscillations are reduced and the curve is better defined.

Considering two experimental runs, it is possible to trace just a line on the plane $F_{d}$. However, combining all the possible couples of tests in order to obtain different lines, the plane $F_{d}$ is better described and a more accurate fitting is possible as shown in Figure 9. 

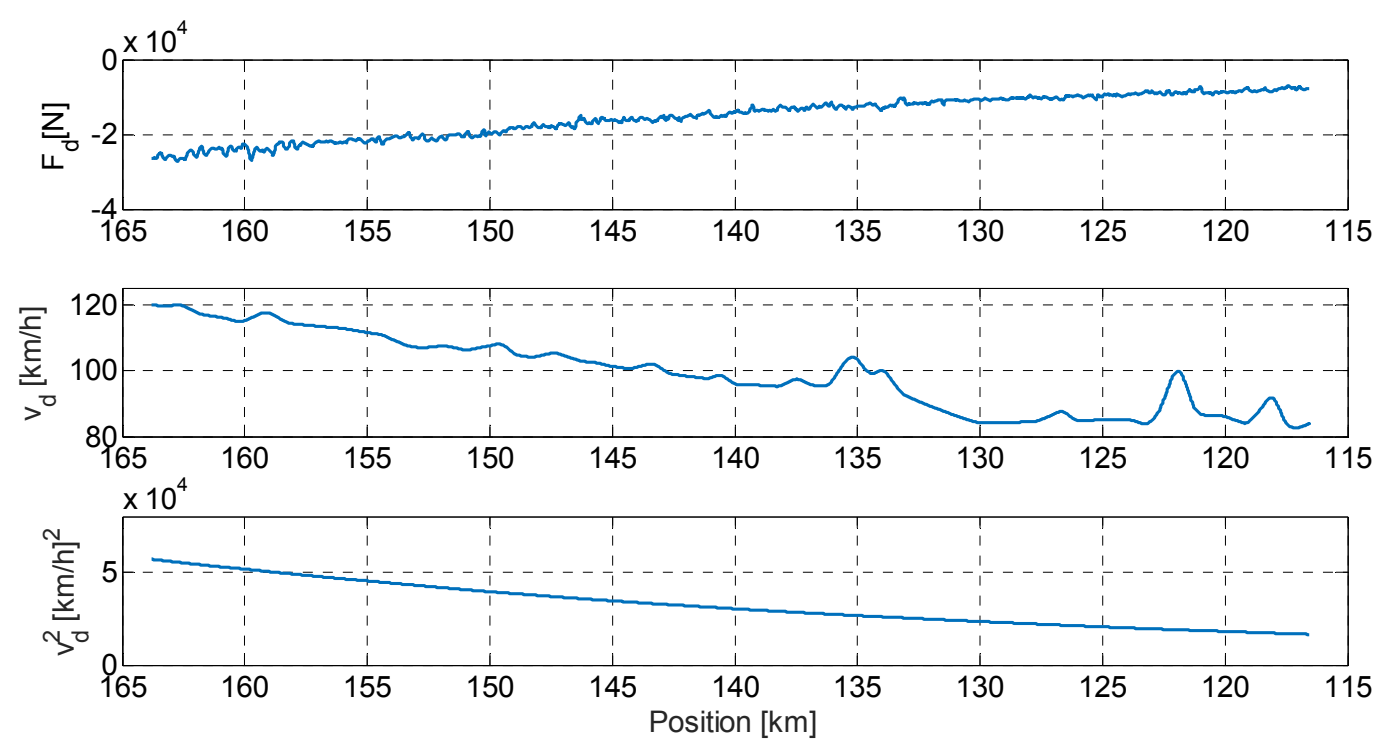

Figure 8: Resistance force $F_{d}, v_{d}$ and $v_{d_{-} q}^{2}$ as functions of the position using the data of the runs 1 and 4 .

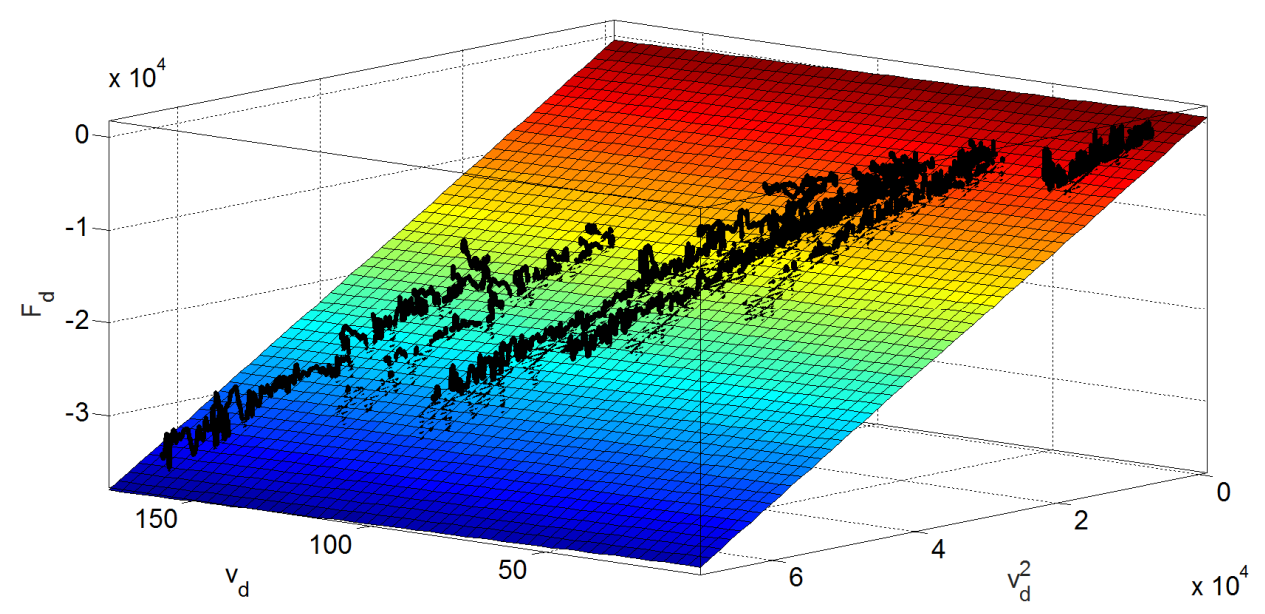

Figure 9: Interpolation of the resistance force $F_{d}, v_{d}$ and $v_{d_{-} q}^{2}$ as functions of the position using the data of the runs 1 and 4 .

The $\mathrm{F}_{d}$ resulting from this interpolation is described by the following equation:

$$
F_{d}=-21.91 \cdot v_{d}-6.21 \cdot v_{d_{-} q}^{2}
$$

\subsubsection{Comparison between the different methods}

The results achived with the four methods are the following:

- regression method:

$$
F_{r}(m, v)=-5.138 \cdot 10^{-3} \cdot m-17.04 \cdot v-6.36 \cdot v^{2}
$$


- speed history identification method:

$$
F_{r}(m, v)=-5.138 \cdot 10^{-3} \cdot m-22.9 \cdot v-6.26 \cdot v^{2}
$$

- regression method on the speed history identification method data:

$$
F_{r}(m, v)=-5.138 \cdot 10^{-3} \cdot m-18.64 \cdot v-6.22 \cdot v^{2}
$$

- POLIMI differential method:

$$
F_{r}(\mathrm{~m}, \mathrm{v})=-5.138 \cdot 10^{-3} \cdot \mathrm{m}-21.91 \cdot \mathrm{v}-6.21 \cdot \mathrm{v}^{2}
$$

The $C$ coefficients estimations are very similar while the estimate of $B$ shows a higher dispersion. The different resistance forces evaluated with the four methods in the speed range of $0-300 \mathrm{~km} / \mathrm{h}$ are reported in Figure 10 as well as the errors evaluated with respect to the results obtained with the third method (combination of regression and speed history method) considered as the reference one. It is possible to see that the error in the evaluation of the resistance force is lower than $2 \%$ for all the methods and, for the POLIMI method, the error is a few higher than $1 \%$.
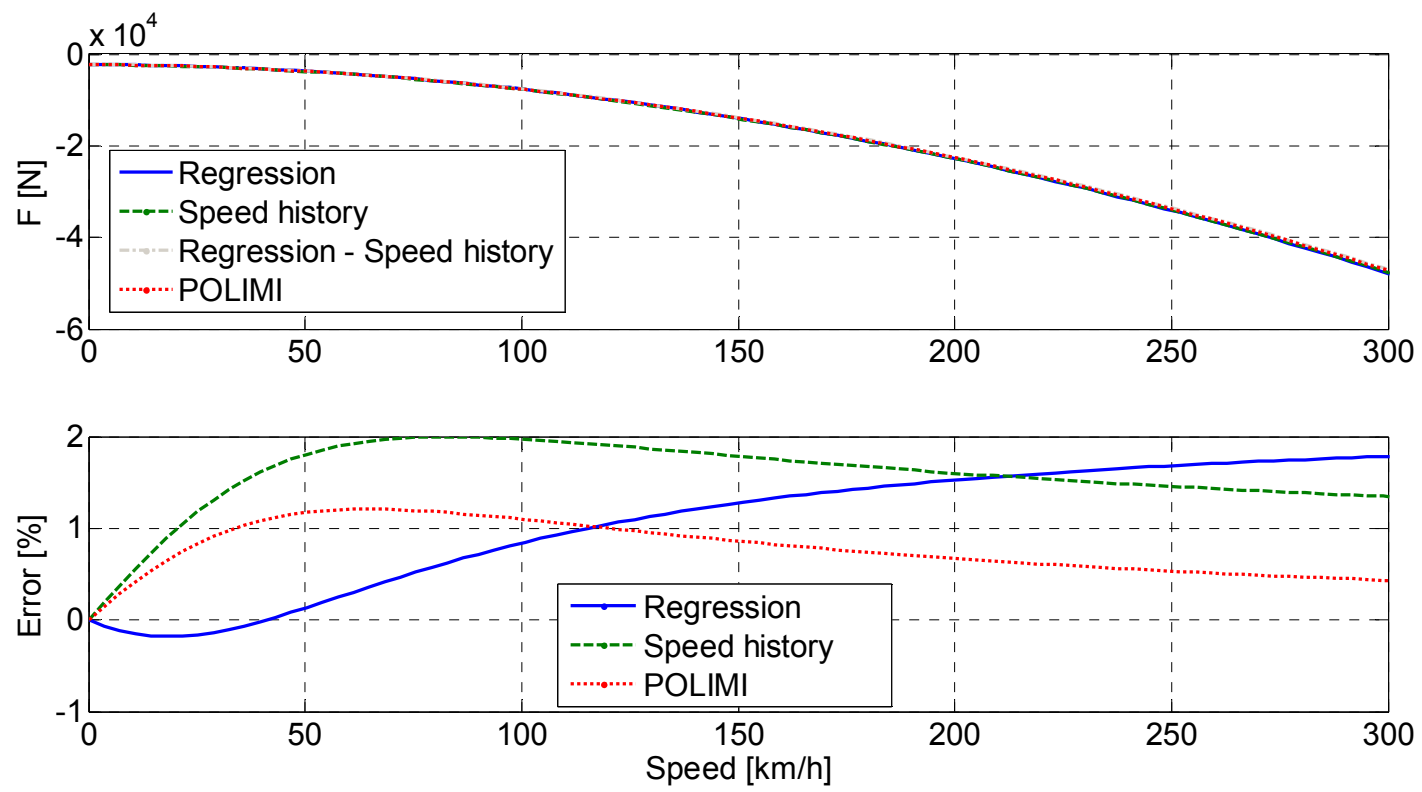

Figure 10: Comparison between the resistance force estimated by the four methods (above) and relative error with respect to the reference method (combination of regression and speed history method) (below).

Table 6 compares the four different methods applied in this paper in terms of:

- input data needed;

- capacity of take into account variations in the air density;

- time demanding. 


\begin{tabular}{|c|c|c|c|c|c|}
\hline & $\underset{\text { coefficient }}{A}$ & $\begin{array}{l}\text { Railway line } \\
\text { characteristics }\end{array}$ & $\underset{\text { Air }}{\text { density }}$ & Other & $\begin{array}{l}\text { Time } \\
1 \text { fast / } \\
5 \text { slow }\end{array}$ \\
\hline Regression & $\begin{array}{l}\text { Has to be } \\
\text { known }\end{array}$ & $\begin{array}{l}\text { Have to be } \\
\text { known }\end{array}$ & Neglected & $\begin{array}{l}\text { It is necessary } \\
\text { to remove the } \\
\text { section with } \\
\text { variable slope } \\
\text { and radius }\end{array}$ & 1 \\
\hline $\begin{array}{l}\text { Speed history } \\
\text { identification }\end{array}$ & $\begin{array}{l}\text { Has to be } \\
\text { known }\end{array}$ & $\begin{array}{l}\text { Have to be } \\
\text { known }\end{array}$ & Considered & $\begin{array}{l}\text { Average the } \\
\text { results is not } \\
\text { accurate }\end{array}$ & 4 \\
\hline $\begin{array}{l}\text { Regression } \\
\text { Speed history }\end{array}$ & $\begin{array}{l}\text { Has to be } \\
\text { known }\end{array}$ & $\begin{array}{l}\text { Have to be } \\
\text { known }\end{array}$ & Considered & & 5 \\
\hline POLIMI & Useless & Useless & Neglected & & 1 \\
\hline
\end{tabular}

Table 6: Comparison between the four methods for the identification of the B and C parameters.

From these results it can be concluded that:

- the regression method, which does not account for the air density variation and which considers a lower quantity of data (no curves nor slopes) leads to results very similar to those found with more expensive methods (speed history and combination of regression and speed history);

- the POLIMI method allows to obtain results equivalent to that found with the other methods prescribed by the CEN standard but with the advantage that it is unnecessary to know the line characteristics and the results do not depend on the estimation of the parameter $A$. As a consequence, a higher dispersion in the A evaluation (CEN requires an uncertainty lower than $10 \%$ for this parameter) do not influence the estimate of the other two parameters $B$ and $C$.

- the variations of the air density lower than $2 \%$, such as those measured in the considered tests, have not a valuable impact on the parameters estimation.

\section{Conclusions}

In conclusion, in this paper the standard approaches for the evaluation of the Davis coefficients are compared to new methods. In particular, it is shown that the three coefficients of the Davis formula as well as the mass factor $\mathrm{k}$ can be estimated only by two tests:

1. coasting tests at very low speed over a high sloping section;

2. coasting tests over a straight and level section starting from the maximum train speed. 
As regards the coefficient $A$, a first estimation of the parameter (named $\hat{A}$ ), is carried out from the data measured with the test 1, neglecting the effects of the terms depending on the vehicle speed, without the need of performing the hauling test as proposed by the CEN standard. The correct estimation of $A$, accounting for the $B$ and $\mathrm{C}$ effects, is then performed with the data of the test 2.

As regards the evaluation of the coefficients $B$ and $C$, four different methods are compared: regression method and speed history method as proposed by the CEN standard, a combination of these two methods and a new method named POLIMI differential method. It is shown that the coefficients estimated with the four approaches are very similar and the errors evaluated with respect to the results obtained with the reference method (combination of regression and speed history method) are lower than $2 \%$ for all the considered methods. On the other hand, the new method proposed by POLIMI presents the following advantages:

- it is unnecessary to know the line characteristics;

- the results do not depend on the estimation of the parameter $A$;

- it is very low time consuming.

The only disadvantage with respect to the speed history method is that it does not account for the effect of the air density but, on the other hand, it has been also shown that variations in this parameter lower than $2 \%$, such as those measured in the considered tests, have not a valuable impact on the coefficients estimation.

\section{References}

[1] Davis Jr., W.J., "The tractive resistance of electric locomotives and cars", Gen. Electr. Rev, 29, 2-24, 1926

[2] Hara, T., Ohkushi, J., Nishimura, B., "Aerodynamic drag of trains”, Q. Rep. RTRI, 8 (4), 226-229, 1967

[3] Guiheu, C., "Resistance to forward movement of TGV-PSE trainsets: evaluation of studies and results of measurements", French Railw. Rev, 1 (1), 13-26, 1983

[4] Peters, J.-L., "Measurement of the influence of tunnel length on the tunnel drag of the ICEIV train". Aerodynamics and ventilation of vehicle tunnels, pp. 739-756, 1991

[5] Report ERRI C, 179/RP 9, Utrecht, April, 1993

[6] Rochard, B.P., Schmid, F., "A review of methods to measure and calculate train resistances", Proceedings of the Institution of Mechanical Engineers, Part F: Journal of Rail and Rapid Transit, 214(4), 185-199, 2000

[7] Lukaszewicz, P., "A simple method to determine train running resistance from full-scale measurements", Proceedings of the Institution of Mechanical Engineers, Part F: Journal of Rail and Rapid Transit, 221(3), 331-337, 2007

[8] Lukaszewicz P., "Running resistance - Results and analysis of full-scale tests with passenger and freight trains in Sweden", Proceedings of the Institution of Mechanical Engineers, Part F: Journal of Rail and Rapid Transit, 221 (2), 183193, 2007 
[9] EN 14067-4 (2005) - A1 (2009). Railway applications - Aerodynamics - Part 4: Requirements and test procedures for aerodynamics on open track 